\title{
Validation of the Publication of New Names and New Combinations Previously Effectively Published Outside the IJSB
}

\author{
List No. $6^{\dagger}$
}

The purpose of this announcement is to effect the valid publication of the following new names and new combinations under the procedure described previously (Int. J. Syst. Bacteriol. 27(3):iv, 1977). Authors and other individuals wishing to have new names and/or combinations included in future lists should send the pertinent reprint or a photocopy thereof to the Editor of the IJSB for confirmation that all of the other requirements for valid publication have been met. It should be noted that the date of valid publication of these new names and combinations is the date of publication of this list, not the date of the original publication of the names and combinations. The authors of the new names and combinations are as given below, and these authors' names will be included in the Author Index of the present issue and in the volume Author Index in this issue of the IJSB.

\begin{tabular}{|c|c|c|c|c|c|c|}
\hline \multirow[b]{2}{*}{ Name } & \multirow[b]{2}{*}{ Proposed as } & \multirow[b]{2}{*}{ Author(s) } & \multirow[b]{2}{*}{ Basonym } & \multicolumn{2}{|c|}{$\begin{array}{l}\text { Literature } \\
\text { citation(s) to: }\end{array}$} & \multirow[b]{2}{*}{ Nomenclatural type $^{a}$} \\
\hline & & & & $\begin{array}{l}\text { Proposal } \\
\text { of name }\end{array}$ & $\begin{array}{l}\text { Descrip- } \\
\text { tion of } \\
\text { the taxon }\end{array}$ & \\
\hline Aeromonas sobria & New species & $\begin{array}{l}\text { Popoff and } \\
\text { Véron }\end{array}$ & & 29 & 29 & $\begin{array}{l}\text { Strain } 208(=\mathrm{CIP} \\
7433)^{b}\end{array}$ \\
\hline $\begin{array}{l}\text { Actinomadura ferru- } \\
\text { ginea }\end{array}$ & New species & Meyer & & 25 & 25 & Strain IMET $9567^{b}$ \\
\hline $\begin{array}{l}\text { Actinomadura liban- } \\
\text { otica }\end{array}$ & New species & Meyer & & 25 & 25 & Strain IMET $9616^{b}$ \\
\hline $\begin{array}{l}\text { Actinomadura spir- } \\
\text { alis }\end{array}$ & New species & Meyer & & 25 & 25 & Strain IMET $9621^{b}$ \\
\hline $\begin{array}{l}\text { Alteromonas putrefa- } \\
\text { ciens }^{c}\end{array}$ & New species & Lee et al. & & 20 & 20 & $\begin{array}{l}\text { Strains NCIB } 10471 \text { (= } \\
\text { ATCC } 8071=\text { Ham- } \\
\text { mer 95) }\end{array}$ \\
\hline Azomonotrichon & New genus & $\begin{array}{l}\text { Thompson } \\
\text { and Sker- } \\
\text { man }\end{array}$ & & 35 & 35 & $\begin{array}{l}\text { A. macrocytogenes } \\
\text { (Jensen) Thompson } \\
\text { and Skerman }\end{array}$ \\
\hline $\begin{array}{l}\text { Azomonotrichon ma. } \\
\text { crocytogenes }\end{array}$ & $\begin{array}{l}\text { New combi- } \\
\text { nation }\end{array}$ & $\begin{array}{l}\text { Thompson } \\
\text { and Sker- } \\
\text { man }\end{array}$ & $\begin{array}{l}\text { Azotobacter ma- } \\
\text { crocytogenes } \\
\text { Jensen }\end{array}$ & 35 & 35 & $\begin{array}{l}\text { Strain } 0 \text { of Jensen }(= \\
\text { ATCC } 12335=\text { NCIB } \\
8700=\text { WR-111) }\end{array}$ \\
\hline Azorhizophilus & New genus & $\begin{array}{l}\text { Thompson } \\
\text { and Sker- } \\
\text { man }\end{array}$ & & 35 & 35 & $\begin{array}{l}\text { A. paspali (Döbereiner) } \\
\text { Thompson and Sker- } \\
\text { man }^{b}\end{array}$ \\
\hline $\begin{array}{l}\text { Azorhizophilus pas- } \\
\quad \text { pali }\end{array}$ & $\begin{array}{l}\text { New combi- } \\
\text { nation }\end{array}$ & $\begin{array}{l}\text { Thomspon } \\
\text { and Sker- } \\
\text { man }\end{array}$ & $\begin{array}{l}\text { Azotobacter } \\
\text { paspali } \\
\text { Döbereiner }\end{array}$ & 35 & 35 & $\begin{array}{l}\text { Strain Ax-8 of Döberei- } \\
\text { ner (= ATCC } 23833= \\
\text { WR-129) }^{b}\end{array}$ \\
\hline $\begin{array}{l}\text { Azotobacter armeni. } \\
\text { acus }\end{array}$ & New species & $\begin{array}{l}\text { Thompson } \\
\text { and Sker- } \\
\text { man }\end{array}$ & & 35 & 35 & $\begin{array}{l}\text { Strain N28 of Kirako- } \\
\text { syan }(=\text { WR-136) }\end{array}$ \\
\hline $\begin{array}{l}\text { Azotobacter nigri- } \\
\text { cans subsp. ach- } \\
\text { romogenes }\end{array}$ & $\begin{array}{l}\text { New subspe- } \\
\quad \text { cies }\end{array}$ & $\begin{array}{l}\text { Thompson } \\
\text { and Sker- } \\
\text { man }\end{array}$ & & 35 & 14 & $\begin{array}{l}\text { Strain A-2 of Jensen (= } \\
\quad \text { WR-41) }\end{array}$ \\
\hline Bacillus schlegelii & $\begin{array}{l}\text { New subspe- } \\
\text { cies }\end{array}$ & $\begin{array}{l}\text { Schenk and } \\
\text { Aragno }\end{array}$ & & 34 & 34 & $\underset{2000)^{b}}{\text { Strain MA } 48 \text { (= DSM }}$ \\
\hline $\begin{array}{l}\text { Beijerinckia indica } \\
\text { subsp. lacticogenes }\end{array}$ & $\begin{array}{l}\text { New subspe- } \\
\text { cies }\end{array}$ & $\begin{array}{l}\text { Thompson } \\
\text { and Sker- } \\
\text { man }\end{array}$ & & 35 & 18 & $\begin{array}{l}\text { Strain WR-119 }(= \\
\quad \text { ATCC 19361) }\end{array}$ \\
\hline $\begin{array}{l}\text { Beijerinckia derxia } \\
\text { subsp. venezuelae }\end{array}$ & $\begin{array}{l}\text { New subspe- } \\
\quad \text { cies }\end{array}$ & $\begin{array}{l}\text { Thompson } \\
\text { and Sker- } \\
\text { man }\end{array}$ & & 35 & 23 & $\begin{array}{l}\text { Strain } 2 \text { of Materassi (= } \\
\quad \text { WR-222) }^{d}\end{array}$ \\
\hline
\end{tabular}

† Lists 1 through 5 were published in the Int. J. Syst. Bacteriol. 27:306, 1977; 29:79, 1979; 29:436, 1979; 30:601, 1980; and 30:676, 1980, respectively. 
TABLE-Continued

\begin{tabular}{|c|c|c|c|c|c|c|}
\hline \multirow[b]{2}{*}{ Name } & \multirow[b]{2}{*}{ Proposed as } & \multirow[b]{2}{*}{ Author(s) } & \multirow[b]{2}{*}{ Basonym } & \multicolumn{2}{|c|}{$\begin{array}{l}\text { Literature } \\
\text { citation(s) to: }\end{array}$} & \multirow[b]{2}{*}{ Nomenclatural type ${ }^{a}$} \\
\hline & & & & $\begin{array}{l}\text { Proposal } \\
\text { of name }\end{array}$ & $\begin{array}{l}\text { Descrip- } \\
\text { tion of } \\
\text { the taxon }\end{array}$ & \\
\hline $\begin{array}{l}\text { Brevibacterium io- } \\
\quad \text { dinum }^{c}\end{array}$ & New species & Collins et al. & & 10 & 10 & Strain NCDO $613^{b}$ \\
\hline $\begin{array}{l}\text { Chromobacterium } \\
\text { fluviatile }\end{array}$ & New species & Moss et al. & & 26 & 26 & $\underset{11159)^{b}}{\text { Strain } 165}(=$ NCTC \\
\hline $\begin{array}{l}\text { Clostridium puni. } \\
\text { ceum }\end{array}$ & New species & Lund et al. & & 22 & 22 & $\begin{array}{l}\text { Strain BL } 70 / 20(= \\
\quad{\text { NCIB } 11596)^{b}}^{b}\end{array}$ \\
\hline $\begin{array}{l}\text { Edwardsiella hosh- } \\
\text { inae }\end{array}$ & New species & $\begin{array}{l}\text { Grimont et } \\
\text { al. }\end{array}$ & & 12 & 12 & $\begin{array}{l}\text { Strain } 2-78(=\text { ATCC } \\
33379=\text { CIP } 78.56)^{b}\end{array}$ \\
\hline $\begin{array}{l}\text { Lactobacillus amylo- } \\
\text { philus }\end{array}$ & New species & $\begin{array}{l}\text { Nakamura } \\
\text { and Crow- } \\
\text { ell }\end{array}$ & & 27 & 27 & Strain NRRL B- $4437^{b}$ \\
\hline Methanobacteriales & New order & $\begin{array}{l}\text { Balch and } \\
\text { Wolfe }\end{array}$ & & 1 & 1 & $\begin{array}{l}\text { Methanobacteriaceae } \\
\text { Barker }\end{array}$ \\
\hline $\begin{array}{l}\text { Methanobacterium } \\
\text { bryantii }\end{array}$ & New species & $\begin{array}{l}\text { Balch and } \\
\text { Wolfe }\end{array}$ & & 1 & 1 & $\begin{array}{l}\text { Strain M.o.H. (= DSM } \\
863)^{b}\end{array}$ \\
\hline Methanobrevibacter & New genus & $\begin{array}{l}\text { Balch and } \\
\text { Wolfe }\end{array}$ & & 1 & 1 & $\begin{array}{l}\text { M. ruminantium (Smith } \\
\text { and Hungate) Balch } \\
\text { and Wolfe }{ }^{b}\end{array}$ \\
\hline $\begin{array}{c}\text { Methanobrevibacter } \\
\text { arboriphilicus ( } M . \\
\text { arboriphilus [sic]) }\end{array}$ & $\begin{array}{l}\text { New combi- } \\
\text { nation }\end{array}$ & $\begin{array}{l}\text { Balch and } \\
\text { Wolfe }\end{array}$ & $\begin{array}{l}\text { Methanobacte- } \\
\text { rium arbo- } \\
\text { philicum } \\
\text { (sic) Zeikus } \\
\text { and Henning }\end{array}$ & 1 & 1 & $\begin{array}{l}\text { Strain DH1 (= DSM } \\
1_{1125)^{b}}^{b}\end{array}$ \\
\hline $\begin{array}{l}\text { Methanobrevibacter } \\
\text { ruminantium }\end{array}$ & $\begin{array}{l}\text { New combi- } \\
\text { nation }\end{array}$ & $\begin{array}{l}\text { Balch and } \\
\text { Wolfe }\end{array}$ & $\begin{array}{l}\text { Methanobacte- } \\
\text { rium rumi- } \\
\text { nantium } \\
\text { Smith and } \\
\text { Hungate }\end{array}$ & 1 & 1 & $\underset{1093)^{b}}{\text { Strain M1 }}(=$ DSM \\
\hline $\begin{array}{l}\text { Methanobrevibacter } \\
\text { smithii }\end{array}$ & New species & $\begin{array}{l}\text { Balch and } \\
\text { Wolfe }\end{array}$ & & 1 & 1 & $\underset{861)^{b}}{\text { Strain PS }(=\text { DSM }}$ \\
\hline Methanococcaceae & New family & $\begin{array}{l}\text { Balch and } \\
\text { Wolfe }\end{array}$ & & 1 & 1 & $\begin{array}{l}\text { Methanococcus Kluyver } \\
\text { and van } \text { Niel }^{b}\end{array}$ \\
\hline Methanococcales & New order & $\begin{array}{l}\text { Balch and } \\
\text { Wolfe }\end{array}$ & & 1 & 1 & $\begin{array}{c}\text { Methanococcaceae } \\
\text { Balch and Wolfe }\end{array}$ \\
\hline $\begin{array}{l}\text { Methanococcus vol- } \\
\quad \text { tae }\end{array}$ & New species & $\begin{array}{l}\text { Balch and } \\
\text { Wolfe }\end{array}$ & & 1 & 1 & 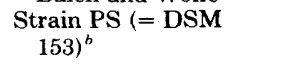 \\
\hline Methanogenium & New genus & $\begin{array}{l}\text { Romesser et } \\
\text { al. }\end{array}$ & & 29 & 29 & $\begin{array}{l}\text { M. cariaci Romesser et } \\
\quad \text { al. }{ }^{b}\end{array}$ \\
\hline $\begin{array}{l}\text { Methanogenium car. } \\
\quad \text { iaci }\end{array}$ & New species & $\begin{array}{l}\text { Romesser et } \\
\text { al. }\end{array}$ & & 29 & 29 & $\underset{1497)^{b}}{\text { Strain JR1 (= DSM }}$ \\
\hline $\begin{array}{l}\text { Methanogenium mar- } \\
\text { isnigri }\end{array}$ & New species & $\begin{array}{l}\text { Romesser et } \\
\text { al. }\end{array}$ & & 29 & 29 & $\begin{array}{c}\text { Strain JR1 ( } \\
1498)^{b}\end{array}$ \\
\hline $\begin{array}{l}\text { Methanomicrobi } \\
\text { aceae }\end{array}$ & New family & $\begin{array}{l}\text { Balch and } \\
\text { Wolfe }\end{array}$ & & 1 & 1 & $\begin{array}{l}\text { Methanomicrobium } \\
\text { Balch and Wolfe }\end{array}$ \\
\hline Methanomicrobiales & New order & $\begin{array}{l}\text { Balch and } \\
\text { Wolfe }\end{array}$ & & 1 & 1 & $\begin{array}{l}\text { Methanomicrobiaceae } \\
\text { Balch and Wolfe }\end{array}$ \\
\hline Methanomicrobium & New genus & $\begin{array}{l}\text { Balch and } \\
\text { Wolfe }\end{array}$ & & 1 & 1 & $\begin{array}{l}\text { M. mobile (Paynter and } \\
\text { Hungate) Balch and } \\
\text { Wolfe }^{b}\end{array}$ \\
\hline $\begin{array}{l}\text { Methanomicrobium } \\
\text { mobile }\end{array}$ & $\begin{array}{l}\text { New combi- } \\
\text { nation }\end{array}$ & $\begin{array}{l}\text { Balch and } \\
\text { Wolfe }\end{array}$ & $\begin{array}{l}\text { Methanobacte- } \\
\text { rium mobile } \\
\text { Paynter and } \\
\text { Hungate }\end{array}$ & 1 & 1 & $\underset{1539)^{b}}{\text { Strain BP }}(=$ DSM \\
\hline Methanosarcinaceae & New family & $\begin{array}{l}\text { Balch and } \\
\text { Wolfe }\end{array}$ & & 1 & 1 & $\begin{array}{l}\text { Methanosarcina Kluy- } \\
\text { ver and van } \mathrm{Niel}^{b}\end{array}$ \\
\hline $\begin{array}{l}\text { Pseudomonas corru- } \\
\text { gata }\end{array}$ & New species & $\begin{array}{l}\text { Roberts and } \\
\text { Scarlett }\end{array}$ & & 33 & 33 & Strain NCPPB $2445^{b}$ \\
\hline $\begin{array}{l}\text { Rhodopseudomonas } \\
\text { blastica }\end{array}$ & New species & $\begin{array}{l}\text { Eckersley } \\
\text { and Dow }\end{array}$ & & 11 & 11 & Strain NCIB $11576^{b}$ \\
\hline Serratia ficaria & New species & $\begin{array}{l}\text { Grimont et } \\
\text { al. }\end{array}$ & & 13 & 13 & $\begin{array}{l}\text { Strain } 4024(=\text { ATCC } \\
33105=\text { CIP } 79.23= \\
\text { ICPB } 4050)^{b}\end{array}$ \\
\hline $\begin{array}{l}\text { Thermoactinomyces } \\
\text { intermedius }\end{array}$ & New species & Kurup et al. & & 19 & 19 & $\begin{array}{l}\text { Strain T-323 }(=\text { ATCC } \\
\quad 33205)^{b}\end{array}$ \\
\hline
\end{tabular}


TABLE-Continued

\begin{tabular}{|c|c|c|c|c|c|c|}
\hline \multirow[b]{2}{*}{ Name } & \multirow[b]{2}{*}{ Proposed as } & \multirow[b]{2}{*}{ Author(s) } & \multirow[b]{2}{*}{ Basonym } & \multicolumn{2}{|c|}{$\begin{array}{l}\text { Literature } \\
\text { citation(s) to: }\end{array}$} & \multirow[b]{2}{*}{ Nomenclatural type ${ }^{a}$} \\
\hline & & & & $\begin{array}{l}\text { Proposal } \\
\text { of name }\end{array}$ & $\begin{array}{l}\text { Descrip- } \\
\text { tion of the } \\
\text { taxon }\end{array}$ & \\
\hline Thermothrix & New genus & $\begin{array}{l}\text { Caldwell et } \\
\text { al. }\end{array}$ & & 9 & 9 & $\begin{array}{l}\text { T. thiopara Caldwell et } \\
\text { al. }^{b}\end{array}$ \\
\hline $\begin{array}{l}\text { Thermothrix thio- } \\
\text { para }\end{array}$ & New species & $\begin{array}{l}\text { Caldwell et } \\
\text { al. }\end{array}$ & & 9 & 9 & Strain ATCC $29244^{e}$ \\
\hline Vibrio campbellii & $\begin{array}{l}\text { New combi- } \\
\text { nation }\end{array}$ & $\begin{array}{l}\text { Baumann et } \\
\text { al. }\end{array}$ & $\begin{array}{l}\text { Beneckea camp- } \\
\quad \text { bellii } \\
\text { Baumann et } \\
\text { al. }\end{array}$ & 3 & 3,4 & Strain ATCC $25920^{b}$ \\
\hline Vibrio fluvialis & New species & Lee et al. & & 21 & 21 & $\begin{array}{l}\text { Strain VL } 5125(= \\
{\text { NCTC } 11327)^{b}}^{b}\end{array}$ \\
\hline Vibrio gazogenes & $\begin{array}{l}\text { New combi- } \\
\text { nation }\end{array}$ & $\begin{array}{l}\text { Baumann et } \\
\text { al. }\end{array}$ & $\begin{array}{l}\text { Beneckea gazo- } \\
\quad \text { genes } \\
\text { Harwood et } \\
\text { al. }\end{array}$ & 3 & $3,16,17$ & $\underset{29988)^{b}}{\text { Strain PB1 }}(=$ ATCC \\
\hline Vibrio harveyi & $\begin{array}{l}\text { New combi- } \\
\text { nation }\end{array}$ & $\begin{array}{l}\text { Baumann et } \\
\text { al. }\end{array}$ & $\begin{array}{l}\text { Beneckea harv } \\
\quad \text { eyi } \\
\text { Reichelt and } \\
\text { Baumann }\end{array}$ & 3 & $3,15,30$ & Strain ATCC $14126^{b}$ \\
\hline Vibrio logei & $\begin{array}{l}\text { New combi- } \\
\text { nation }\end{array}$ & $\begin{array}{l}\text { Baumann et } \\
\text { al. }\end{array}$ & $\begin{array}{l}\text { Photobacterium } \\
\quad \text { logei } \\
\text { Harwood et } \\
\text { al. }\end{array}$ & 3 & $2,3,17$ & $\begin{array}{l}\text { Strain } 584 \\
29985)^{b}\end{array}$ \\
\hline Vibrio natriegens & $\begin{array}{l}\text { New combi- } \\
\text { nation }\end{array}$ & $\begin{array}{l}\text { Baumann et } \\
\text { al. }\end{array}$ & $\begin{array}{l}\text { Beneckea na- } \\
\quad \text { triegens } \\
\text { Baumann et } \\
\text { al. }\end{array}$ & 3 & $3,4,28$ & Strain ATCC $14048^{b}$ \\
\hline Vibrio nereis & $\begin{array}{l}\text { New combi- } \\
\text { nation }\end{array}$ & $\begin{array}{l}\text { Baumann et } \\
\text { al. }\end{array}$ & $\begin{array}{l}\text { Beneckea ner- } \\
\quad \text { eida } \\
\text { Harwood et } \\
\text { al. }\end{array}$ & 3 & $3,4,15$ & $\underset{25917)^{b}}{\operatorname{Strain} 80}(=$ ATCC \\
\hline $\begin{array}{l}\text { Vibrio nigripulchri- } \\
\text { tudo }\end{array}$ & $\begin{array}{l}\text { New combi- } \\
\text { nation }\end{array}$ & $\begin{array}{l}\text { Baumann et } \\
\text { al. }\end{array}$ & $\begin{array}{l}\text { Beneckea nigra- } \\
\text { pulchrituda } \\
\text { Baumann et } \\
\text { al. }\end{array}$ & 3 & 3,5 & Strain ATCC $27043^{b}$ \\
\hline Vibrio pelagius & $\begin{array}{l}\text { New combi- } \\
\text { nation }\end{array}$ & $\begin{array}{l}\text { Baumann et } \\
\text { al. }\end{array}$ & $\begin{array}{l}\text { Beneckea pela. } \\
\quad \text { gia } \\
\text { Baumann et } \\
\text { al. }\end{array}$ & 3 & 3,4 & Strain ATCC $25916^{b}$ \\
\hline Vibrio splendidus & $\begin{array}{l}\text { New combi- } \\
\text { nation }\end{array}$ & $\begin{array}{l}\text { Baumann et } \\
\text { al. }\end{array}$ & $\begin{array}{l}\text { Beneckea splen- } \\
\quad \text { dida } \\
\text { Reichelt et al. }\end{array}$ & 3 & $3,6,31$ & $\begin{array}{l}\text { Strain ATCC } 33125(= \\
\text { NCMB 1) }\end{array}$ \\
\hline Yersinia frederiksenii & New species & Ursing et al. & & 36 & 36 & 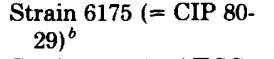 \\
\hline Yersinia intermedia & New species & $\begin{array}{l}\text { Brenner et } \\
\text { al. }\end{array}$ & & 8 & 8 & $\begin{array}{l}\text { Strain } 3953(=\text { ATCC } \\
29909=\text { Bottone } 48= \\
\text { Chester } 48=\text { CIP 80- } \\
28)^{b}\end{array}$ \\
\hline Yersinia kristensenii & New species & $\begin{array}{l}\text { Bercovier et } \\
\text { al. }\end{array}$ & & 7 & 7 & $\begin{array}{l}\text { Strain } 105 \text { (= CIP 80- } \\
\left.3^{30}\right)^{b}\end{array}$ \\
\hline
\end{tabular}

${ }^{a}$ Abbreviations: ATCC, American Type Culture Collection, Rockville, Md.; CIP Collection de l'Institut Pasteur, Paris, France; DSM, Deutsche Sammlung von Mikroorganismen, Göttingen, Germany; ICPB, International Collection of Phytopathogenic Bacteria, University of California, Davis, Calif.; IMET, Institute for Microbiology and Experimental Therapy, Jena, East Germany; NCDO, National Collection of Dairy Organisms, Reading, England; NCIB, National Collection of Industrial Bacteria, Aberdeen, Scotland; NCMB, National Collection of Marine Bacteria, Aberdeen, Scotland; NCPPB, National Collection of Plant Pathogenic Bacteria, Harpenden, England; NCTC, National Collection of Type Cultures, London, England; NRRL, Northern Utilization Research and Development Division, USDA, Peoria, ILL.; WR, Wheat Research Institute, Toowoomba, Queensland, Australia.

${ }^{b}$ Type designated by the author(s).

c Proposed as a new combination; however, the basonym on which the new combination was based was not validly published; hence, the name is cited here as proposed as a new species rather than as a new combination.

$d$ Type designated by the author(s) in a personal communication to the Editor, IJSB; this announcement establishes the type and satisfies the requirement for valid publication.

'Type by monotypy. 


\section{LITERATURE CITED}

1. Balch, W. E., G. E. Fox, L. J. Magrum, C. R. Woese, and R. S. Wolfe. 1979. Methanogens: reevaluation of a unique biological group. Microbiol. Rev. 43:260-296.

2. Bang, S. S., P. Baumann, and K. H. Nealson. 1978. Phenotypic characterization of Photobacterium logei (sp. nov.), a species related to $P$. fischeri. Curr. Microbiol. 1:285-288.

3. Baumann, P., L. Baumann, S. S. Bang, and M. J. Woolkalis. 1980. Reevaluation of the taxonomy of Vibrio, Beneckea, and Photobacterium: abolition of the genus Beneckea. Curr. Microbiol. 4:127-132.

4. Baumann, P., L. Baumann, and M. Mandel. 1971. Taxonomy of marine bacteria: the genus Beneckea. J. Bacteriol. 107 :268-294

5. Baumann, P., L. Baumann, M. Mandel, and R. D. Allen. 1971. Taxonomy of marine bacteria: Beneckea nigrapulchrituda sp. n. J. Bacteriol. 108:1380-1383.

6. Beijerinck, M. W. 1900. On different forms of hereditary variation in microbes. Proc. K. ned. Akad. Wet. 3:352365.

7. Bercovier, H., J. Ursing, D. J. Brenner, A. G. Steigerwalt, G. R. Fanning, G. P. Carter, and H. H. Mollaret. 1980. Yersinia kristensenii: a new species of Enterobacteriaceae composed of sucrose-negative strains (formerly called atypical Yersinia enterocolitica or Yersinia enterocolitica-like). Curr. Microbiol. 4:219224.

8. Brenner, D. J., H. Bercovier, J. Ursing, J. M. Alonso, A. G. Steigerwalt, G. R. Fanning, G. P. Carter, and H. H. Mollaret. 1980. Yersinia intermedia: a new species of Enterobacteriaceae composed of rhamnosepositive, melibiose-positive, raffinose-positive strains (formerly called Yersinia enterocolitica or Yersinia enterocolitica-like). Curr. Microbiol. 4:207-212.

9. Caldwell, D. E., S. J. Caldwell, and J. P. Laycock. 1976. Thermothrix thiparus gen. et sp. nov. a facultatively anaerobic facultative chemolithotroph living at neutral $\mathrm{pH}$ and high temperature. Can. J. Microbiol. 22:1509-1517.

10. Collins, M. D, D. Jones, R. M. Keddie, and P. H. A. Sneath. 1980. Reclassification of Chromobacterium io. dinum (Davis) in a redefined genus Brevibacterium (Breed) as Brevibacterium iodinum nom. rev.; comb. nov. J. Gen. Microbiol. 120:1-10.

11. Eckersley, K., and C. S. Dow. 1980. Rhodopseudomonas blastica sp. nov.: a member of the Rhodospirillaceae. J. Gen. Microbiol. 119:465-473.

12. Grimont, P. A. D., F. Grimont, C. Richard, and R. Sakazaki. 1980. Edwardsiella hoshinae, a new species of Enterobacteriaceae. Curr. Microbiol. 4:347-351.

13. Grimont, P. A. D., F. Grimont, and M. P. Starr. 1979. Serratia ficaria sp. nov., a bacterial species associated with Smyrna figs and the fig wasp Blastophaga psenes. Curr. Microbiol. 2:277-282.

14. Jensen, V., and E. J. Petersen. 1954. Studies on the occurrence of Azotobacter in Danish forest soils. K. Vet-Landbohojsk. Ársskr. 1954:95-108.

15. Johnson, F. H., and I. V. Shunk. 1936. An interesting new species of luminous bacteria. J. Bacteriol. 31:585592.

16. Harwood, C. S. 1978. Beneckea gazogenes sp. nov., a red, facultatively anaerobic, marine bacterium. Curr. Microbiol. 1:233-238.

17. Harwood, C. S., S. S. Bang, P. Baumann, and K. H. Nealson. 1980. Photobacterium logei sp. nov., nom. rev.; Beneckea nereida sp. nov., nom. rev.; and Beneckea gazogenes sp. nov., nom. rev. Int. J. Syst. Bacteriol. 30:655.

18. Kauffmann $Y$., and $P$. Toussaint. 1951. Un noveau genre fixateur de l'azote atmospherique: Azotobacter lacticagenes. C. R. Acad. Sci., Paris 223:710-711.

19. Kurup, V. P., G. E. Hollick, and E. F. Pagan. 1980. Thermoactinomyces intermedius, a new species of amylase negative thermophilic actinomycetes. ScienceCiencia. Bol. Cien. Sur 7:104-108.

20. Lee, J. V., D. M. Gibson, and J. M. Shewan. 1977. A numerical taxonomic study of some Pseudomonas-like marine bacteria. J. Gen. Microbiol. 98:439-451.

21. Lee, J. V., P. Shread, A. L. Furniss, and T. N. Bryant. 1981. Taxonomy and description of Vibrio fluvialis sp. nov. (synonym group F vibrios, group EF6). J. Appl. Bacteriol. 50:73-95.

22. Lund, B. M., T. F. Brocklehurst, and G. M. Wyatt. 1981. Characterization of strains of Clostridium puniceum sp. nov., a pink-pigmented, pectolytic bacterium. J. Gen. Microbiol. 122:17-26.

23. Materassi, R., G. Florenzano, W. Balloni, and F. Favilli. 1966. Su una nuova specie di Beijerinckia (Beijerinckia venezuelae n. sp.) isolata da terreni venezuelani. Ann. Microbiol. Enzimol. 16:201-215.

24. Merkel, J. R., E. D. Tranganza, B. B. Mukherjee, T. B. Griffin, and J. M. Prescott. 1964. Proteolytic activity and general characteristics of a marine bacterium, Aeromonas proteolytica sp. n. J. Bacteriol. 87:12271233.

25. Meyer, J. 1979. New species of the genus Actinomadura. Z. Allgem. Mikrobiol. 19:37-44.

26. Moss, M. O., C. Ryall, and N. A. Logan. 1978. The classification and characterization of chromobacteria from a lowland river. J. Gen. Microbiol. 105:11-21.

27. Nakamura, L. K., and C. D. Crowell. 1979. Lactobacil lus amylophilus, a new starch-hydrolyzing species from swine waste-corn fermentation. Dev. Ind. Microbiol. 20: $532-540$.

28. Payne, W. J., R. G. Eagon, and A. K. Williams. 1961 Some observations on the physiology of Pseudomonas natriegens nov. spec. Ant. van Leeuwenhoek J. Microbiol. Serol. 27:121-128.

29. Popoff, M., and M. Véron. 1976. A taxonomic study of the Aeromonas hydrophila-Aeromonas punctata Group. J. Gen. Microbiol. 94:11-22.

30. Reichelt, J. L., and P. Baumann. 1973. Taxonomy of the marine, luminous bacteria. Arch. Mikrobiol. 94: 283-330.

31. Reichelt, J. L., P. Baumann, and L. Baumann. 1976. Study of genetic relationships among marine species of the genera Beneckea and Photobacterium. Arch. Mikrobiol. 110:101-120.

32. Romesser, J. A., R. S. Wolfe, F. Mayer, E. Spiess, and A. Walther-Mauruschat. 1979. Methanogenium, a new genus of marine methanogenic bacteria, and characterization of Methanogenium cariaci sp. nov. and Methanogenium marisnigri sp. nov. Arch. Mikrobiol. 121:147-153.

33. Scarlett, C. M., J. T. Fletcher, P. Roberts, and R. A. Lelliott. 1978. Tomato pith necrosis caused by Pseudomonas corrugata $\mathrm{n}$. sp. Ann. Appl. Biol. 88:105-114.

34. Schenk, A., and M. Aragno. 1979. Bacillus schlegelii, a new species of thermophilic, facultatively chemolithoautotrophic bacterium oxidizing molecular hydrogen. J. Gen. Microbiol. 115:333-341.

35. Thompson, J. P., and V. B. D. Skerman. 1980. Azotobacteraceae: the taxonomy and ecology of the aerobic nitrogen-fixing bacteria. Academic Press, London, 405 pp.

36. Ursing, J., D. J. Brenner, H. Bercovier, G. R. Fanning, A. G. Steigerwalt, J. Brault, and H. H. Mollaret. 1980. Yersinia frederiksenii: a new species of Enterobacteriaceae composed of rhamnose-positive strains (formerly called atypical Yersinia enterocolitica or Yersinia enterocolitica-like). Curr. Microbiol. 4:213217. 